\title{
Mechanisms of rumination change in adolescent depression (RuMeChange): study protocol for a randomised controlled trial of rumination-focused cognitive behavioural therapy to reduce ruminative habit and risk of depressive relapse in high- ruminating adolescents
}

Henrietta Roberts ${ }^{1}$, Rachel H. Jacobs ${ }^{2}$, Katie L. Bessette ${ }^{3}$, Sheila E. Crowell ${ }^{4}$, Mindy Westlund-Schreiner ${ }^{3}$, Leah Thomas ${ }^{3}$, Rebecca E. Easter ${ }^{3}$, Stephanie L. Pocius ${ }^{3}$, Alina Dillahunt ${ }^{3}$, Summer Frandsen ${ }^{3}$, Briana Schubert ${ }^{3}$, Brian Farstead ${ }^{3}$, Patricia Kerig ${ }^{4}$, Robert C. Welsh ${ }^{3}$, David Jago ${ }^{1}$, Scott A. Langenecker ${ }^{3}$ and Edward R. Watkins ${ }^{1 *}$ (D)

\begin{abstract}
Background: Adolescent-onset depression often results in a chronic and recurrent course, and is associated with worse outcomes relative to adult-onset depression. Targeting habitual depressive rumination, a specific known risk factor for relapse, may improve clinical outcomes for adolescents who have experienced a depressive episode. Randomized controlled trials (RCTs) thus far have demonstrated that rumination-focused cognitive behavioral therapy (RFCBT) reduces depressive symptoms and relapse rates in patients with residual depression and adolescents and young adults with elevated rumination. This was also observed in a pilot RCT of adolescents at risk for depressive relapse. Rumination can be measured at the self-report, behavioral, and neural levels- using patterns of connectivity between the Default Mode Network (DMN) and Cognitive Control Network (CCN). Disrupted connectivity is a putative important mechanism for understanding reduced rumination via RFCBT. A feasibility trial in adolescents found that reductions in connectivity between DMN and CCN regions following RFCBT were correlated with change in rumination and depressive symptoms.
\end{abstract}

(Continued on next page)

\footnotetext{
* Correspondence: E.R.Watkins@exeter.ac.uk

'Mood Disorders Centre, School of Psychology, Sir Henry Wellcome Building for Mood Disorders Research, University of Exeter, Exeter EX4 4LN, UK

Full list of author information is available at the end of the article
}

C C The Author(s). 2021 Open Access This article is licensed under a Creative Commons Attribution 4.0 International License, which permits use, sharing, adaptation, distribution and reproduction in any medium or format, as long as you give appropriate credit to the original author(s) and the source, provide a link to the Creative Commons licence, and indicate if changes were made. The images or other third party material in this article are included in the article's Creative Commons licence, unless indicated otherwise in a credit line to the material. If material is not included in the article's Creative Commons licence and your intended use is not permitted by statutory regulation or exceeds the permitted use, you will need to obtain permission directly from the copyright holder. To view a copy of this licence, visit http://creativecommons.org/licenses/by/4.0/. The Creative Commons Public Domain Dedication waiver (http://creativecommons.org/publicdomain/zero/1.0/) applies to the data made available in this article, unless otherwise stated in a credit line to the data. 


\begin{abstract}
(Continued from previous page)
Method: This is a phase III two-arm, two-stage, RCT of depression prevention. The trial tests whether RFCBT reduces identified risk factors for depressive relapse (rumination, patterns of neural connectivity, and depressive symptoms) in adolescents with partially or fully remitted depression and elevated rumination. In the first stage, RFCBT is compared to treatment as usual within the community. In the second stage, the comparator condition is relaxation therapy. Primary outcomes will be (a) reductions in depressive rumination, assessed using the Rumination Response Scale, and (b) reductions in resting state functional magnetic resonance imaging connectivity of DMN (posterior cingulate cortex) to CCN (inferior frontal gyrus), at 16 weeks post-randomization. Secondary outcomes include change in symptoms of depression following treatment, recurrence of depression over 12 months post-intervention period, and whether engagement with therapy homework (as a dose measure) is related to changes in the primary outcomes.

Discussion: RFCBT will be evaluated as a putative preventive therapy to reduce the risk of depressive relapse in adolescents, and influence the identified self-report, behavioral, and neural mechanisms of change.

Understanding mechanisms that underlie change in rumination is necessary to improve and further disseminate preventive interventions.
\end{abstract}

Trial registration: ClinicalTrials.gov Identifier: NCT03859297, registered 01 March 2019.

Keywords: Rumination, Depression, Adolescence, Cognitive behavioral therapy, Development, Resting state networks

\section{Background}

Depression is a leading cause of disability, affecting more than 264 million people worldwide [1,2]. The prevalence of depression has increased by nearly $20 \%$ in the last decade. Notably, the greatest increase in incidence for depression is during adolescence and early adulthood. In addition, $25 \%$ of individuals experience their first depressive episode before the age of 19 [1-4]. Despite the availability of well-established treatment options (e.g., cognitive behavioral therapy, CBT; antidepressant medications), significant challenges in the prevention and treatment of depression remain. There is considerable evidence indicating that depression is sub-optimally treated by existing psychosocial and pharmaceutical interventions (e.g., [5-7]), with a 40-60\% remission rate. Of those in remission, $30-50 \%$ experience residual symptoms at the end of treatment [8-10], with high rates of relapse. A recent meta-analysis of adults reported that $29 \%$ of patients experienced relapse within 1 year and 56\% experienced a recurrence within 2 years [11], with similar statistics in adolescents [12], emphasizing that for many, depression has a chronic recurrent course.

Adolescence is a key developmental window in which to intervene with individuals who are vulnerable to depressive episodes [13], as depressive illness has cumulative educational, psychosocial, occupational, and economic burden. Moreover, adolescent-onset depression typically follows a chronic, recurrent course, and is associated with more negative outcomes relative to adult-onset depression [14-21]. Evidence also exists that the recurrent form of depression tends to worsen as individuals transition into adulthood, with more severe and longer episodes [22]. However, current gold-standard treatments do not successfully treat a substantial number of adolescents (30$60 \%)$ and are not effective in reducing risk of relapse [12].
As a consequence, there is a rapidly expanding literature investigating interventions to reduce depression in adolescence and emerging adulthood, and to prevent relapse and recurrence in this age group [23]. Finally, adolescence is a period in which healthy habit development might achieve sustained benefits.

One proposed means to reduce depression recurrence in young people is to use preventive interventions that target specific proximal risk factors that have an established role in the onset, maintenance, and recurrence of depression. Meta-analyses have reported that whereas universal preventive interventions (aimed at whole populations) achieve only small-to-non significant effect sizes [24], targeted interventions (aimed at individuals presenting with subclinical symptoms and/or established risk factors) have larger reductions in hazard ratios, and these effects are sustained over a longer period [25, 26]. Within the framework of these proximal risk factors, there is an increased appreciation that the mechanisms underlying increased risk for psychopathology may be dimensional in nature, and may even be transdiagnostic [27-29]. For example, the Research Domain Criteria (RDoC) framework by the National Institute of Mental Health (NIMH) proposes dimensional risks for illness can exist along a continuum and may not be specific to a given disorder [27, 28]. Novel interventions should therefore seek to alter such mechanisms in order to effectively target the emergence and maintenance of mental health difficulties such as depression. Building on this framework, prevention mechanism trials [30] seek to establish whether interventions can reduce specific risk factors for psychopathology or increase established resilience factors.

Rumination is a known proximal risk factor implicated in the onset, maintenance and relapse of depression, and 
has been identified as a malleable target for intervention $[31,32]$. Prospective longitudinal research has demonstrated that degree of ruminative habit (i.e., the frequency, intensity and automaticity of ruminative thought in response to negative affect) predicts (a) onset and duration of major depressive episodes [33, 34]; (b) depressive symptoms, after controlling for baseline depression and anxiety, across a range of follow-up periods [35, 36]; and (c) slower response to $\mathrm{CBT}$ and antidepressant treatment, as well as reduced likelihood of recovery following treatment $[37,38]$. Degree of ruminative habit also mediates the effects of other identified risk factors on depressive onset [39]. Importantly, rumination is a sensitive, relevant risk factor for adolescent depression and prospectively predicts fluctuations in depressive symptoms over time in this age group [33, 40, 41]. Developmental studies indicate that the ruminative response style emerges and stabilizes during the transition from childhood to adolescence, consolidating into an emotionally pernicious habit that is strongly predictive of psychopathology in adolescence (see [42] for recent review). The emergence of rumination coincides with a period of rapid cognitive and neural development, during which continued, significant structural and functional changes are observed within the cognitive control network $(\mathrm{CCN})$ and increasing connectivity within the default mode network (DMN) [42-48]. It has been suggested that increasing coherence within these brain networks may be important to understanding the emergence of rumination as a habitual response style during this developmental window [42].

Rumination has been related to abnormal functioning of key nodes and networks of the brain. From resting state and mood induction studies, evidence is present that rumination is associated with elevated activation and connectivity between DMN nodes (e.g., [49]), increased connectivity between $\mathrm{CCN}$ and DMN, and reduced connectivity within $\mathrm{CCN}$. The DMN is thought to support self-referential processing, passive waiting, and attention to the internal environment. Both resting state-functional MRI (rs-fMRI) and task-based approaches have been employed to study the neural correlates of rumination among adults, and to a lesser extent, adolescents with depression [45, 49-58]. The temporal correlation of blood-oxygen level dependent (BOLD) activation, and perhaps synchronization and engagement, of specified nodes of the brain are reflected in rs-fMRI measures. Self-reported rumination is related to activation in regions of the DMN during task-based studies, including the dorsomedial prefrontal cortex (dmPFC), posterior cingulate cortex (PCC), and also visual and somatosensory areas. Alternatively, activation in the superior frontal gyrus in the $\mathrm{CCN}$ has been found during rumination induction tasks $[48,50,52]$. The $\mathrm{CCN}$ is a distinct network of distributed neural nodes that broadly supports integrative executive functions such as inhibitory control, working memory, and sustained complex attention [59]. One function of inhibitory control is its ability to modulate negative affective processes, including rumination [46, 60, 61]. Consistent with a CCN disruption hypothesis of elevated rumination, rs-fMRI studies have reported that disrupted/elevated DMN-CCN connectivity (and decreased within $\mathrm{CCN}$ connectivity) is associated with increased rumination and decreased inhibitory control [46, 61, 62]. Moreover, behavioral disruption is evident in the context of induced rumination in the laboratory, with more errors and greater interference (slower response times) observed during a concurrent attentional task [48, 63]. As such, the maladaptive effects of rumination can be observed at both the clinical level, in elevated risk for depression, and at the neural and behavioral level, including impaired performance on cognitive tasks and altered functioning of key brain networks.

A recent systematic review examined whether treatments for depression that specifically target rumination produce better outcomes than more generic treatments that do not target rumination [64]. Results showed that treatments specifically targeting rumination had significantly larger effect sizes in reducing rumination than other treatments. These effects on rumination post-treatment were significantly associated with reductions in depression severity only in variants of cognitive-behavioral therapy specifically designed to target rumination such as rumination-focused CBT (RFCBT) [64]. RFCBT differentiates between functional and dysfunctional styles of repetitive thinking; the more adaptive thinking style is conceptualized as being concrete and specific, whereas the unhelpful thinking style is abstract and evaluative, consistent with the phenomenology of depressive rumination. RFCBT conceptualizes ruminative thinking as an unhelpful habitual response and focuses on the use of functional analysis of rumination. Thus, RFCBT combines behavioral activation strategies with novel techniques to foster a concrete, process-focused style of thinking. In support of this model, an RCT comparing treatment-as-usual (TAU, including antidepressant medication) versus TAU plus RFCBT in patients with medication-refractory residual depression found that the addition of RFCBT reduced depressive symptoms more than medication alone [65]. RFCBT was found to improve remission rates $(62 \%$ vs. $21 \%)$ and reduce relapse rates $(10 \%$ vs. $53 \%)$ at 6 months post-treatment, with changes in rumination scores found to be a significant mediator of these effects. A further independent trial found that RFCBT improved depressive symptoms and reduced rumination, relative to a waiting list control group, in patients with residual depression [66]. More recently, RFCBT has been evaluated in adolescents and young people and as a preventive treatment for youth at increased risk for depression with promising results. In a high-risk preventive 
design, group and internet RFCBT were compared to a waiting list control condition in a large sample of Dutch adolescents and young adults with elevated rumination, but without current depression. Relative to the waiting list control, both RFCBT interventions significantly reduced rumination and depression at post-intervention and oneyear follow-up, and halved the one-year incidence rates of depression [67]. In another preventive study, guided online RFCBT significantly reduced the subsequent onset of major depression, relative to a usual practice control, in UK undergraduates with elevated rumination, especially for those young people reporting higher levels of stress at baseline [68].

Importantly, a recent pilot RCT compared 8 weeks of TAU+RFCBT to TAU+assessment in 33 adolescents with a history of major depression who were at risk of relapse and observed that RFCBT significantly reduced rumination and depression relative to the TAU control group [69]. fMRI scans pre- and post-intervention showed that adolescents who received RFCBT demonstrated significant decreases in rs-fMRI connectivity between regions of DMN (the left posterior cingulate cortex) and the CCN (the right inferior frontal gyrus and bilateral inferior temporal gyri). The degree of change in connectivity was correlated with changes in self-reported depression and rumination, suggesting that the DMN and $\mathrm{CCN}$ may begin to function more independently (or in less conflict) as rumination reduces. RFCBT therefore is a promising candidate intervention for the prevention of depressive relapse and recurrence in adolescents following prior depressive episode(s), and may improve longer term outcomes for this at-risk population.

These findings indicate that RFCBT is able to modify rumination in adolescents, and shows potential as a targeted intervention to reduce risk of depressive relapse in high-risk adolescents. However, no full-scale study has evaluated RFCBT adapted for adolescents at risk of depressive relapse. Moreover, research has not yet revealed the neural mechanisms underlying increases or decreases in rumination, either in the moment or over time. This knowledge regarding the neural processes affecting rumination is particularly pertinent during the critical developmental period of adolescence, when ruminative habits typically emerge.

\section{Aims and objectives}

The primary aim of this phase III, two-stage prevention mechanism randomised controlled trial is to evaluate whether RFCBT is effective in reducing the primary outcomes of (a) self-reported rumination and (b) rs-fMRI connectivity between regions of the DMN and $\mathrm{CCN}$, when compared to a TAU + assessment-only (AO) control arm in the first stage (NIMH R61 grant phase) (assessments performed within context of the study and
TAU provided in community), assessed at primary endpoint of 16-20 weeks post-baseline. These indices are chosen as potential predictors of risk for future depression; that is, this operates as a targeted trial of a prevention mechanism [30, 70]. The second stage (potential NIMH R33 grant phase if Go targets are met in first stage) includes comparison of RFCBT to relaxation therapy on the same primary outcomes. Secondary outcomes include change in symptoms of depression at 16 weeks, recurrence of depression over 12 months post-intervention period, and whether engagement with therapy homework (as a dose measure) is related to changes in the primary outcomes.

\section{Methods}

\section{Study design}

The study consists of a two-arm parallel-group singleblind randomised controlled trial. Stage 1 compares RFCBT to TAU + AO; stage 2 compares RFCBT to TAU + relaxation. The assessment team members will be blind to treatment arm. Our primary hypotheses are that RFCBT will be superior to the control arm in reducing (a) rumination, and (b) rs-fMRI connectivity between DMN and CCN. Our secondary hypotheses are that (a) that RFCBT will be superior to the control arm in reducing recurrence of depression over 12 months; and (b) engagement with RFCBT homework (dose response) will be related to changes in (i) rumination, (ii) DMN and CCN resting state connectivity, and (iii) degree of change in regional brain activation during a rumination induction from pre- to post- treatment. The study design was published in the National Clinical Trials Website (NCT03859297) in March 2019. The only substantive change is the move to tele-assessment and teletherapy in March 2020 in response to the COVID-19 pandemic. The neuroimaging protocol was discontinued from March through June 2020, and then reinstated with COVID-19 safety precautions.

\section{Study setting}

The study takes place at the University of Utah in the United States of America. Participants attend assessment appointments at the Huntsman Mental Health Institute (formerly University Neuropsychiatric Institute) on the University of Utah campus, which provides primary inpatient and outpatient psychiatric services to young people and adults who are primarily residents throughout the Salt Lake Valley and Intermountain West region.

\section{Eligibility criteria}

Eligible participants will be postpubertal adolescents aged 14-17 with a previous diagnosis of Major Depressive Disorder (MDD), based on the Diagnostic and Statistical Manual (DSM-5) [71] criteria and confirmed by 
assessments using the Kiddie Schedule for Affective Disorders and Schizophrenia - Present and Lifetime Version DSM-5 (KSADS-PL) [72, 73] $(N=60$ in stage 1 and $N=120$ in stage 2). Participants must have been in full or partial remission for a minimum of 2 weeks (typically much longer); partial remission is defined as meeting less than five clinical-threshold symptoms for MDD according to the KSADS-PL. In addition, participants will be required to have elevated levels of rumination, defined as an elevated Rumination Response Scale (RRS) [35]. Age-adjusted RRS threshold cut-offs for males are from 28 to 31 and for females from 35 to 38 ; the minimum cut-off levels increase with participant age [35, 74].

Exclusion criteria include a lifetime history of conduct disorder, autism spectrum disorder, any psychotic disorder (or psychotic episode unexplained by other known medical causes), or bipolar disorder, an estimated intelligence quotient (IQ) of 75 or less as estimated by a computer adaptation of the Synonym Knowledge subtest [75]; elevated current depressive symptoms as indicated by a raw score greater than 45 on the Children's Depression Rating Scale - Revised (CDRS-R) [76]; endorsing suicide attempt or plan within the past 6 months, as assessed by the KSADS-PL, CDRS-R, and Lifetime Suicide Attempt Self-Injury Interview (L-SASI) [77]; reporting anorexia/bulimia or alcohol/substance abuse within the past 6 months. Currently receiving psychotropic medication other than stimulants for ADHD and antidepressants (e.g., anxiolytics and most atypical antipsychotics) will be exclusionary with the exception of those that are prescribed for treating depression only. If taking medications, participants will be required to have been on a stable dose of medication for the past 4 weeks, with no change in specific medication for past 6 weeks. The study will also exclude individuals reporting current or recent (past year) treatment with CBT, or related variants (e.g., detailedoriented structured therapy involving elements such as cognitive restructuring, homework, or a CBT focus), and those reporting prior treatment with rumination focus. Due to residual concerns from institutional review boards about the brain imaging protocol, individuals who are currently pregnant are excluded. For safety and data quality reasons, those who have MRI contraindications including non-removable metal braces, tattoos with metal, or claustrophobia are excluded. In instances where potential participants either decline consent or provide consent but do not meet eligibility requirements, we will offer a list of clinically relevant treatment resources outside of the study, including the option of receiving the same treatment in outpatient clinic from one of the investigators. We will keep information collected from participants who consented and were later deemed ineligible for the purposes of assessing generalizability and sample representativeness.

\section{Recruitment}

Participants for the proposed study will be recruited from the University of Utah Hospitals and Clinics and from a number of additional sources and organisations (e.g., The Balanced Mind Foundation, National Alliance on Mental Illness, American Foundation for Suicide Prevention), via advertisements on local radio stations (e.g., KSL radio), in schools, on social media (e.g. Facebook, NextDoor), and on Utah public transit (between May 2019 and May 2021 (estimated)). Adolescent/young adult-based hospitals and clinics, as well as high schools and universities in the Salt Lake City area, will be locations targeted for additional recruitment. Our successful retention rates with pilot work (approximately 83\% over 2 years [69]) will be bolstered through close contact with all study participants and close monitoring at weekly research team meetings. If obstacles to study recruitment and retention are encountered, contingency plans such as increased advertisement, increased resources to cover research assistant time focused on recruitment outreach efforts, announcements to colleagues, and scheduled calls to participants, will be developed to address these challenges in collaboration with the team. Following verification of initial eligibility via telephone screening, participants are scheduled for a baseline visit during which we obtain complete written informed parental consent and adolescent participant assent prior to enrolment in the study. Parents will also be asked to consent to contribute information about the adolescent and relevant family medical history. For adolescents who turn 18-years-old during the course of the study, they will be asked to provide written informed consent to continue in the study once they are aged 18 . Further eligibility will be determined by the research team via administration of the KSADS-PL, CDRS-R, RRS, and Synonyms Knowledge Test at the baseline visit. After the final baseline assessment procedures (diagnostic, neuropsychological, fMRI, and the stress and relaxation session), the patients will be randomly assigned to receive RFCBT or participate in the control group.

\section{Participant timeline}

For further details on participant timeline, see Figs. 1 and 2.

\section{Sample size}

This trial was funded as the first stage (R61 grant) in a potential two-stage study, with the second stage (R33 grant) comparing RFCBT to an active control of relaxation therapy. Moving to the second stage is contingent on the first stage meeting the Go criteria of baseline to post-intervention between-treatment arm effect size changes of .5SD (effect size) in RRS and .5SD (effect size) in rs-fMRI connectivity from left PCC to right IFG. These effect sizes were chosen to reflect a noticeable clinical difference, necessary to progress to second stage. 


\begin{tabular}{|c|c|c|c|c|c|c|c|c|c|c|c|c|c|c|c|c|c|c|c|}
\hline \multirow[b]{2}{*}{ TIMEPOINT } & \multirow{2}{*}{$\begin{array}{l}\text { Screen } \\
t_{3} \\
\end{array}$} & \multirow{2}{*}{$\begin{array}{c}\begin{array}{c}\text { Base- } \\
\text { line } 1 \\
-t_{2}\end{array} \\
\end{array}$} & \multirow{2}{*}{$\begin{array}{c}\text { Base- } \\
\text { line 2 } \\
-t_{1} \\
\end{array}$} & \multirow[t]{2}{*}{$\begin{array}{l}\text { Enroll- } \\
\text { ment }\end{array}$} & \multirow[t]{2}{*}{$\begin{array}{l}\text { Relax- } \\
\text { ation }\end{array}$} & \multicolumn{10}{|c|}{ Weekly treatment sessions } & \multirow{2}{*}{$\begin{array}{c}\begin{array}{c}\text { Mid } \\
\text { Treat- } \\
\text { Tent }\end{array} \\
t_{6}\end{array}$} & \multirow{2}{*}{$\begin{array}{c}\text { Post } \\
\text { Treat- } \\
\text { ment } \\
t_{12}\end{array}$} & \multirow{2}{*}{$\begin{array}{c}\begin{array}{c}6 \\
\text { months }\end{array} \\
t_{13}\end{array}$} & \multirow{2}{*}{\begin{tabular}{|c}
12 \\
12 \\
months \\
$t_{14}$ \\
\end{tabular}} \\
\hline & & & & & & $t_{1}$ & $t_{2}$ & $t_{3}$ & $t_{4}$ & $t_{5}$ & $t_{7}$ & $t_{s}$ & $t_{9}$ & $t_{10}$ & $t_{11}$ & & & & \\
\hline \multicolumn{20}{|l|}{ ENROLMENT: } \\
\hline Eligibility screen & $x$ & & & & & & & & & & & & & & & & & & \\
\hline $\begin{array}{r}\begin{array}{r}\text { Informed } \\
\text { assent/consent }\end{array}\end{array}$ & & $x$ & & & & & & & & & & & & & & & & & \\
\hline Allocation & & & $x$ & & & & & & & & & & & & & & & & \\
\hline \multicolumn{20}{|l|}{ INTERVENTION: } \\
\hline RFCBT & & & & & $x$ & + & & & & & & & & & $\vec{a}$ & & & & \\
\hline $\begin{array}{l}\text { Assessment Only } \\
\text { (Stage 1) }\end{array}$ & & & & & $x$ & & & & & & & & & & & & & & \\
\hline Relaxation (Stage & & & & & $x$ & ـ & & & & & & & & & $\rightarrow$ & & & & \\
\hline \multicolumn{20}{|l|}{ ASSESSMENTS: } \\
\hline $\begin{array}{c}\text { Self report } \\
\text { measures }\end{array}$ & & $x$ & & & & $x$ & & $\mathrm{x}$ & & $\mathrm{x}$ & & $x$ & & & $x$ & $x$ & $x$ & $\mathrm{x}$ & $x$ \\
\hline $\begin{array}{l}\text { Diagnostic } \\
\text { Interview }\end{array}$ & & $x$ & & & & & & & & & & & & & & & $x$ & & $x$ \\
\hline $\begin{array}{c}\text { Neuroimaging } \\
\text { assessment }\end{array}$ & & & $x$ & & & & & & & & & & & & & $x$ & $x$ & & \\
\hline Diary measures & & & & & & $x$ & $\mathrm{x}$ & $\mathrm{x}$ & $\mathrm{x}$ & $\mathrm{x}$ & $x$ & $x$ & $\mathrm{x}$ & $x$ & $x$ & & & & \\
\hline $\begin{array}{c}\text { Acceptability } \\
\text { measures }\end{array}$ & & & & & & & & & & & & & & & & & $x$ & $\mathrm{x}$ & $\mathrm{x}$ \\
\hline
\end{tabular}

Fig. 1 RuMeChange schedule of enrolment, interventions, and assessments

For this stage, the target recruitment is $n=30$ per group. Power was computed using gPower 3.1 for a repeated measurements model in the first stage (R61), to compare the effects of RFCBT versus TAU + AO where observations are nested within subjects, targeting a .5SD (effect) size change, and with known reliability of RRS $(r=.77)$ and rs-fMRI connectivity of left PCC to right IFG $(r=$ .71). On this basis, a target recruitment of $n=30$ per treatment arm is estimated to provide power of .99 for RRS and .98 for rs-fMRI. Assuming up to 15\% follow-up attrition at post-intervention follow-up, power remains $>.94$ for both comparisons. For the R33 stage, there will be an active treatment comparator so the effect size difference is likely to be smaller. To achieve adequate power to detect a meaningful clinical difference, we assumed a minimum difference of $0.22 \mathrm{SD}$ for each comparison; for power at 0.80 , alpha at 0.05 , assuming $15 \%$ follow-up attrition, we need to recruit 60 young people per treatment arm $(N=120)$.

\section{Randomisation and allocation concealment}

Block randomization will be performed using Matlab to produce computer-generated random codes that are stratified according to key clinical indicators and demographic variables, including RRS, CDRS, sex and age. Block randomization will be conducted by individuals (RWC, LRT) who will not share rumination scores, be providing therapy, or conducting independent assessments and will therefore be independent of the assessment and treatment staff. After each of four waves, the effect of randomization on age, sex distribution, RRS score, and proportion randomized to each arm are evaluated, and the formula is adjusted to maximize equivalence of the two groups.
The independent evaluators (IEs) who conduct diagnostics at baseline and follow-up visits will have separate calendars and channels of contact from the therapists and coordinators. Assignment of therapist will be based upon mutual alignment of schedules of the adolescent and therapist. When more than one therapist is available in an aligned slot, therapist caseload and adolescent preference (male or female therapist) will be taken into consideration. IEs who complete evaluations (for presence of mood disorder, mood symptoms) after the assessment period will remain blinded to treatment. We will report the extent to which this is successful, and the proportion of post-treatment evaluations that are conducted by blinded IEs.

IEs will administer the intake baseline assessment, the post-treatment assessment (16-20 weeks following baseline), as well as subsequent 12 month post-intervention follow-up interviews. Between the baseline and posttreatment assessment, adolescents will additionally report bi-weekly RRS measurements that are not shared with the therapist. To avoid accidental unblinding of IEs, a research assistant will meet with the participant and legal guardian prior to each diagnostic evaluation, in order to remind them of the importance of not disclosing this information to their assessor. Fidelity of blinding will be monitored with a blindness questionnairecompleted by IEs during follow-up assessments as noted above.

There is a detailed protocol to address suicidal ideation and risk, in which further questions are used to ascertain risk and appropriate actions are taken including consulting with supervisor, discussing with family, contacting crisis resources, or inpatient hospitalization. The trial also has a trained adolescent psychiatrist as independent study monitor to evaluate and address any safety issues. 


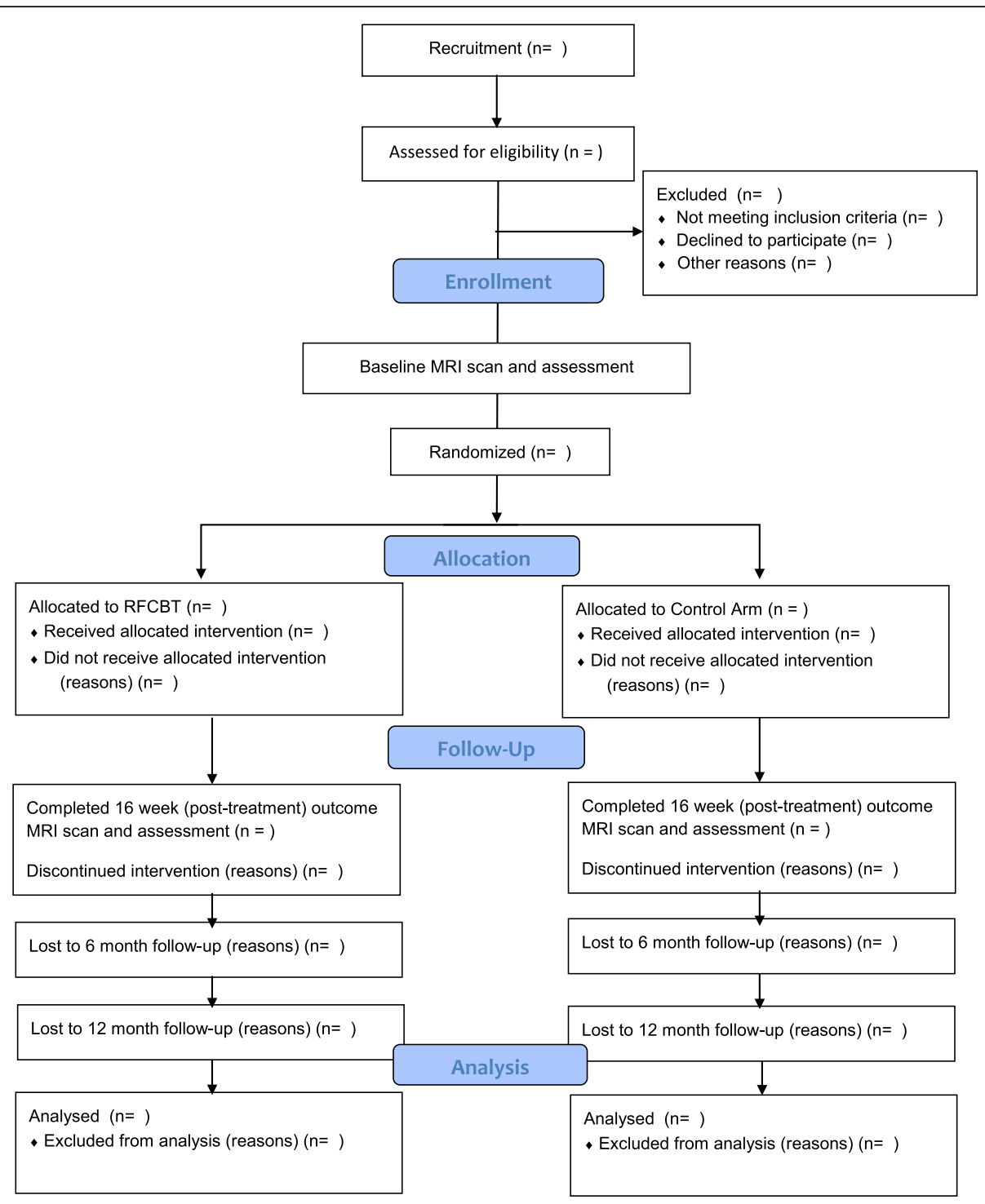

Fig. 2 CONSORT flow diagram for RuMeChange

\section{Interventions}

Following a pilot study [69], remitted MDD adolescents will be randomised to $\mathrm{AO}$ as a comparison to RFCBT (Stage 1 of the trial). This is because RFCBT is not a common treatment in use in clinical settings, and has not previously been used in clinical trials of adolescents with remitted depression. This initial phase will therefore establish that RFCBT is effective in reducing rumination and changing the rsfMRI connectivity of neural networks as predicted in this population. This comparison will additionally provide clinical decision metrics on who will benefit the most from RFCBT, which can be taken forward to refine the treatment protocol for Stage 2, in which RFCBT is compared to relaxation. All participants, regardless of arm, will receive one session of practice in progressive muscle relaxation, before progressing to RFCBT or AO.
Adolescents randomised to RFCBT will meet with a therapist on a typically weekly basis for 45-60 min following the manualized intervention for a target of 10 to 14 sessions [78], typically over a 3 month period. Rumination is characterized by abstract thinking about the causes, meanings, and implications of symptoms and difficulties, as exemplified by asking "Why me?" questions, with this abstract thinking implicated in the negative consequences of rumination. In contrast, encouraging more concrete thinking, focused on how events happen and the context and sequence of what did happen can help an individual move into a specific action and plan, keep an event in perspective, and reduce depression. RFCBT targets rumination and other maladaptive forms of emotion regulation such as suppression and avoidance and provides skills training in effectively coping with 
rumination. RFCBT specifically addresses rumination through psychoeducation, adopting a functional analytic approach to the learned habitual behaviour of rumination, and a focus on shifting processing style [79-81]. The adolescent is taught to notice triggers for rumination as well as the consequences of rumination and how to shift into practicing a more adaptive strategy such as an attention training exercise, behavioural activation, thinking in a concrete way, self-compassion, or active problem-solving [79-81]. RFCBT directly teaches adolescents to recognize rumination and to notice the influence this behavior has on their mood. The acronym ASK is used to teach adolescents that, rather than ruminating, which tends to take the form of passive, abstract, and critical forms of thinking about oneself, to be Active, Specific, and Kind. Through regular functional analyses in sessions, adolescents learn about their cycle of emotions and how the habit of rumination can make it harder to take action. A key skill taught is to change abstract and general thinking about difficulties and problems into a more specific, concrete and contextualized approach. Patients are taught to spot their abstract overgeneralized thoughts and "Why?" questions and to replace these with more helpful "How?" questions. Consistent with changing a habit, adolescents repeatedly practice using these different techniques in response to their warning signs for rumination (IF-THEN strategic plans) [82, 83].

Trial therapists will be psychologists and masters-level therapists who have completed a minimum of 5 days of training in RFCBT with EW and RHJ, (the treatment author and PI of the pilot adolescent trial), over 4 separate sessions, and will receive weekly supervision sessions from the treatment author and expert supervisors throughout the intervention delivery, including direct audio or video review of sessions. Therapists will use individual session templates to record and track therapy content and ensure adherence to the treatment protocol (including session checklists and overall therapy adherence module checklists). Digital recordings of therapy sessions will be used for the purposes of clinical supervision and monitoring. Twenty percent of sessions will be randomly reviewed using an adapted version of the Cognitive Therapy Rating Scale [84], modified for the specific principles and techniques of RFCBT, by an independent rater blind to treatment progress in order to monitor fidelity.

Participants will be provided with paper handouts and electronic tablet devices that are set-up with access to the therapy and homework materials. These (or the individuals' cell phones) will additionally deliver personalised prompts to complete an electronic therapy diary (through the PACO Survey application), in order to monitor homework practice and track the relationships between practice of therapy techniques and levels of rumination in everyday life.

TAU may occur during the course of the trial with the participant's primary care physician, mental health service provider, or psychiatrist, including a referral resource list that is provided to every participant for emergency and outpatient mental health services. TAU received will be assessed at the follow-up assessments. The primary treatments provided in this age range are expected to be supportive therapy and medication management (we expect roughly half will be on stable medication).

In the second (R33) stage of the trial, relaxation training will occur parallel to RFCBT to control for the effects of therapy structure, time, therapist contact, use of an active coping strategy for mood modulation, and attention. As such, sessions will occur weekly for 45-60 min and will include progressive muscle relaxation, simple breathing techniques, and guided imagery that focus on bodily and somatic relaxation. Adolescents randomized to relaxation will also receive exercises to do as homework in between sessions, parallel to the RFCBT group. This includes adaptions of relaxation formats for different situations that may not allow for a full execution (e.g., an airplane runway, in an office chair). Given that relaxation techniques are empirically supported for alleviating distress, anxiety, and depression among many different populations, we expect some effect of relaxation but it does not specifically target the mechanism of rumination.

\section{Outcomes}

Outcomes will be assessed from baseline (pre-randomisation) to $16-20$ weeks and 12 months post-intervention (see Fig. 1).

\section{Primary outcome}

The primary treatment outcome is self-reported reductions in rumination, as assessed using the brooding scale of the RRS [36] from baseline to 16-20 weeks postbaseline. Brooding is defined as "passive and judgmental pondering of one's mood" ([85], p. 99), and is associated with maladaptive strategies and depression. We will use the Reliable Change Index (RCI) as an indication of change in brooding rumination scores [86]. RCI is a measure of how much change in a measurement can be ascribed as a meaningful movement at a statistical level, for the individual case, based upon the error inmeasurement, reliability of an instrument, and moderating for the effects of regression to the mean. In pilot work with adolescents, $57 \%$ of those treated with RFCBT obtained a change in total rumination scores at or above an RCI level, suggesting an adequate dose of targeted therapy (vs. 14\% of AO group, e.g., random reduction). 
The primary brain target (capturing the neural mechanisms compensating for rumination as a result of RFCBT) is elevated connectivity between the DMN and $\mathrm{CCN}$ (and diminished within- $\mathrm{CCN}$ connectivity) during rs-fMRI $[46,87]$. Resting state fMRI (rs-fMRI) will investigate concurrent (or synchronized) changes in blood flow while adolescents are at rest with eyes open looking at a fixation cross. All adolescents will complete two identical MRI scans: at baseline and then at postintervention (16 weeks), corresponding to the end of RFCBT sessions. A 64-channel coil will be used, and rsfMRI sequences will be consistent with the Adolescent Brain Cognitive Development (ABCD) study protocol, with same TR, TE, and multiband sequences. We will collect 4 rs-fMRI runs at each of the two scans. Seed-based analyses will be used to probe changes to functional connectivity. The primary neural outcome is therefore reduced rs-fMRI connectivity between the left posterior cingulate cortex and right inferior frontal gyrus (nodes within the DMN and CCN respectively) [57]. Networkbased analysis will also be probed for CCN-DMN changes in connectivity. Two experimental task-based paradigms $[63,88]$ will be employed to investigate directed induced rumination vs. distraction (block design), and uninstructed use of rumination during a sustained attention task [63]. These probes are explicitly designed to look at network function and dynamics of DMN nodes alone and with $\mathrm{CCN}$, and how they may change selectively with RFCBT.

\section{Secondary outcome}

The occurrence and time to onset of any subsequent mood disorder and/or depressive episode are secondary outcomes. Recurrence of MDD and related mood disorders will be assessed by diagnostic interview (KSADS-PL) administered by IEs at post-intervention and at 12 months.

Dose is a rating of homework completion and skill integration (rated by the clinician), and degree of rumination change (defined by RCI). The primary clinical outcome is therefore change on the RRS score (as captured by the RCI) after treatment. We will additionally examine the stability of RRS change at 6 and 12 months post-intervention.

\section{Data collection and management}

To promote participant retention, the study coordinators will keep in contact with the families via their preferred method (e.g., text messaging, emailing, and telephone calls). The participant payment schedule will be spread out across visits, so that adolescents will be compensated for each time they attend an assessment. Follow-up appointments will be scheduled in advance and at the families' convenience, and participants will be contacted before appointments to remind them of the appointment and confirm the date/location. In the event of a missed visit, participants will be contacted through email and telephone. We will then wait until the next assessment/ visit and attempt to schedule the participant. If the participant is unresponsive after several missed visits without a response, they will be called directly by co-PI Langenecker. They will be asked if they wish to discontinue or if no response, they will be considered lost to follow-up. To enhance interest and retention, additional strategies will be employed, including sending a birthday postcard (if agreed), provision of a printed $2 \mathrm{~d}$ picture of the adolescent's brain (after the intervention period, if agreed), and a 3D model of the adolescent's brain (at 12 month follow-up, if agreed).

The MRI data will be stored in mirrored RAID (redundant array of independent disks) arrays, which are both firewall-protected and isolated from access outside the immediate local network. The RAID arrays will also be used to store other data from the participants, and will be backed up daily with digital tape. All non-image subject data will bestored in REDCap. REDCap is a meta-data driven software solution that facilitates secure collaborative data access, real time data validation and auditing [89], and is compliant with the Health Insurance Portability and Accountability Act (HIPAA) and General Data Protection Regulation (GDPR) best practices [90, 91].

All data will be collected, stored and handled in accordance with HIPAA [90]. The study protocol, documents, data management, and safety plans have been approved by the University of Utah Institutional Review Board (IRB_00113733). Approval for sharing and analysis of deidentified and coded data has been obtained by the University of Exeter Psychology Ethics Committee and data sharing plans have been subject to ethical review to ensure these are compliant with the European Union GDPR [91]. It will be made clear to participants how their data will be used and stored prior to obtaining consent to participate in the trial. All participants will be assigned a unique ID; coding all study data and images with an identifying number and referring to this number in all analyses will preserve confidentiality. The participants will not be identified in any reports of behavioural, clinical, or functional data.

Two independent clinicians with expertise in cognitive behavioural therapy and in working with adolescents, as well as an independent statistician at Utah, compose the internal Data Safety and Monitoring Board (DSMB), which will meet in person at least once per year for routine review of records (review of protocol, of a prototypic record, review of all adverse events, and review of all protocol deviations), and at least every 6 months by telephone/videoconference, if there are any actionable items that occur for enrolment or conflict of interest concerns, or participant safety review. The Independent Study Monitor (ISM) (XongRaio, M.D., adolescent psychiatrist), 
in concert with the DSMB, will set up a study charter before enrolment of the first participant that includes enrolment data, safety data, and data integrity. The DSMB will review de-identified study data for data quality and integrity, adherence to the protocol, participant safety, study conduct and progress twice per year. The ISM and DSMB will be blinded to participant group assignment. In the case of specific incidents (expected or unexpected adverse events), the ISM or DSMB may request from the study team information pertaining to group assignment for a given individual, or aggregate information on a group of individuals who experienced an adverse event. The ISM and DSMB retain autonomy to make determinations and recommendations in the case of adverse events. As the treatments have already been used with adolescents in a pilot study and in a larger European trial [66-68], such modifications are not anticipated. The ISM or DSMB will issue a monitoring report to the PI following each review/ meeting, including any significant actions taken and any final recommendation(s) with regard to the study's continuation. The ISM will be responsible for reviewing trial stopping/pausing decisions and enrolment recommendations. The ISM will review, within $24 \mathrm{~h}$, any suicide-related assessments or concerns. In cases with reemerging depression without increased suicidal ideation, the ISM will be consulted after two consecutive elevated measurements and will then decide how the participant shall proceed. The participant may be asked to pause participation, in which cases referrals will be made to suitable clinical services. At such a time as when the adolescent is stable and low risk, they may resume participation in the trial. There are no anticipated stopping rules for the entire study. The intervention is a low-risk intervention in those who are currently low in symptoms, and families can pursue treatment as usual during the trial.

\section{Statistical methods}

Primary analyses will be conducted on intention-to-treat basis (as randomised) and missing data assumed to be missing at least at random (MAR), confirmed using missing data analysis. Unplanned missing data will be handled via multiple imputation (MI). Secondary variables will be used to improve the estimation of missing data. Sensitivity analysis, assuming a variety of MI models (Missing at Random; Missing Not at Random), will confirm the probable impact of missing data.

To compare the effects of RFCBT with the control arm, mixed-effects longitudinal models will be used. The first analysis tests change in RRS scores; the second analysis tests change in connectivity between the left posterior cingulate cortex and right inferior frontal gyrus. For each, the random subject intercept parameter will explain the variation in measurements of subjects at baseline, whereas the random slope parameter will explain variation over time. The models will also incorporate within-subject variation. The group by time interaction parameter will be used to differentiate the trend of RFCBT from that of the control arm on each outcome. Sustainability of improvement will be measured utilizing data from post-intervention to until 12 months post-intervention. To measure relation between degree of daily engagement in homework and degree of reduction in rumination, we will calculate Pearson correlations. A contrast using pre and post measures of rs-fMRI between left PCC and right IFG in adolescents on the RFCBT arm will be compared to the control arm, as described in Jacobs et al. 2016 [57]. The same approach will be adopted for exploratory, task-based fMRI, capturing state rumination.

Preliminary data and published studies include targeted seed-based analyses of connectivity between PCC (DMN node) and left inferior temporal gyrus (CCN node) $[46,47,58,60,62,64-69]$. Connectivity of PCC to inferior frontal gyrus (also $\mathrm{CCN}$ ) was considered. Because rs-fMRI analyses are rapidly evolving, there may be additional analytic strategies that evolve between now and interim study analysis - we will co-publish any use of these new techniques in the same paper with the primary seed-based analysis. We will evaluate and publish a Bayesian technique to allow for subtle variations in seed and node 3D coordinates. This is based upon observed sample to sample fluctuations in spatial smoothing, functional connectomic parcels, such that exact coordinates may not align for seed and node from sample to sample. As an example, our prior work shows similar, and only partially overlapping nodes for identification of PCC to superior frontal gyrus increased connectivity in MDD compared to healthy controls in three independently published samples [46].

Subsequent analyses will use the Complier Average Causal Effect (CACE) analysis [92, 93]. CACE assumes that the effect of treatment depends on the adherence to treatment (dose effects), which in turn is dependent on randomisation, and that randomisation has no direct effect on the outcome variable. As such, CACE takes into account adherence and compliance with the treatment in order to estimate a treatment effect, whilst also retaining the benefits of randomisation. Briefly, CACE estimates the difference in the outcome variable between the compliers in the treatment arm and the compliers in the control arm had they been offered the treatment, assuming that there are similar rates of compliance in both arms as a result of randomisation. Compliance will be defined as attending at least 8 out of a possible maximum of 16 RFCBT sessions.

\section{Discussion}

Adolescent-onset depression often follows a chronic, recurrent course, and is associated with a number of 
long-term negative outcomes [14-21]. Understanding specific treatment mechanisms that can reduce shortand long-term risk for depressive relapse in young people who have experienced a depressive episode is therefore important in order to improve clinical outcomes. RFCBT was developed to specifically target maladaptive rumination, and has been found to be effective in reducing rumination and, subsequently,risk of depressive relapse in clinical trials with adults, and in a recent pilot trial with adolescents. Rumination can be measured at the neural level, in increased within-network DMN connectivity, and patterns of connectivity between DMN and CCN. The current study will use RFBCT in adolescents at risk of depressive relapse, with the goal of achieving both significant clinical change in rumination, and associated changes in connectivity between key DMN and CCN nodes that have been linked to decline in problematic rumination. Enhanced understanding of the neurocognitive mechanisms underpinning rumination and the prevention of depressive relapse could help to develop better targeted personalised intervention strategies.

\begin{abstract}
Abbreviations
AO: Assessment Only; CACE: Complier Average Causal Effect; CDSR: Children's Depression Rating Scale; CBT: Cognitive Behaviour Therapy; CCN: Cognitive Control Network; DSMB: Data Safety and Monitoring Board; DMN: Default Mode Network; DSM-V: Diagnostic and Statistical Manual fifth edition; dmPFC: dorsomedial prefrontal cortex; fMRI: functional magnetic resonance imaging; GDPR: General Data Protection Regulation; HIPAA: Health Insurance Portability and Accountability Act; ISM: Independent Study Monitor; KSADSPL: Kiddie Schedule for Affective Disorders - Present and Lifetime Version; MDD: Major Depressive Disorder; MAR: Missing at least random; MI: Multiple imputation; NDCT: National Database for Clinical Trials; RCl: Reliable Change Index; rs-fMRI: resting-state functional magnetic resonance imaging; RFCBT: Rumination Focused Cognitive Behavioural Therapy; RRS: Rumination Response Scale; TAU: Treatment-as-usual
\end{abstract}

\section{Acknowledgements}

Not applicable.

\section{Authors' contributions}

ERW and SAL obtained funding for the RuMeChange project and designed the trial. EW and SL coordinate the overall trial. HR prepared the first draft of this manusciprt. All authors (HR, RHJ, KLB, SEC, MW-S, LT, REE, SLP, AD, SF, BS, $B F, P K, R C W, D J, S A L, E R W)$ contributed to the writing of the manuscript, edited relevant sections, and approved the final version.

\section{Funding}

This trial is funded by a National Institute of Mental Health (NIMH) R61 award MH118060 to co-Principal Investigators Scott Langenecker (University of Utah) and Edward Watkins (University of Exeter). Developing ruminationfocused treatment to reduce risk for depression recurrence in adolescence (RuMeChange). The study sponsor and funder have no influence on the conduct, delivery or reporting of the research.

\section{Availability of data and materials}

The study is registered on clinicaltrials.gov (NCT03859297), which will be updated with the published protocol and the study results and associated publications. De-identified data and results will be submitted to the National Database for Clinical Trials Related to Mental Illness (NDCT). NDCT provides a secure system to support the submission, sharing and access of relevant data at all levels of biological and behavioral organization and for all data types. Access to data for research purposes will be provided through the Data
Access Committee. In accordance with the trial resource sharing agreement, data will additionally be deposited into the National Database for Autism Research, which is a US national database that houses deidentified data from many federal studies, irrespective of the disease/disorder/condition under study.

\section{Declarations}

\section{Ethics approval and consent to participate}

Ethical and professional guidelines will be followed at all times, in line with Good Clinical Practice guidelines. This study has been reviewed by, and received approval from, the University of Utah Institutional Review Board and the University of Exeter Psychology Ethics Committee. The study is currently using trial protocol version 1.4 dated 8/30/2018. The trial sponsor is University of Utah, Salt Lake City, Utah, United States,

84108. Protocol modifications are recorded and communicated to trial partners, sponsor, review boards and the Data Monitoring and Safety Board and Independent Study Monitor.

Following verification of initial eligibility via telephone screening, participants are scheduled for a baseline visit during which we obtain complete written informed parental consent and adolescent participant assent prior to enrolment in the study. Parents will also be asked to consent to contribute information about the adolescent and relevant family medical history. For adolescents who turn 18-years-old during the course of the study, they will be asked to provide written informed consent to continue in the study once they are aged 18.

\section{Consent for publication}

Not applicable.

\section{Competing interests}

EW developed rumination-focused CBT, and receives royalties from Guilford Press for a published treatment manual for this intervention and receives remuneration for providing training workshops in this intervention. There are no other competing interests.

\section{Author details}

'Mood Disorders Centre, School of Psychology, Sir Henry Wellcome Building for Mood Disorders Research, University of Exeter, Exeter EX4 4LN, UK. ${ }^{2}$ University of Chicago, Chicago, IL 60612, USA. ${ }^{3}$ Department of Psychiatry, University of Utah, Salt Lake City, UT 84108, USA. ${ }^{4}$ Department of Psychology, University of Utah, Salt Lake City, UT 84108, USA.

Received: 20 February 2021 Accepted: 1 April 2021

Published online: 23 April 2021

\section{References}

1. GBD 2017. Disease and Injury Incidence and Prevalence Collaborators. Global, regional, and national incidence, prevalence, and years lived with disability for 354 diseases and injuries for 195 countries and territories, 1990-2017: a systematic analysis for the Global Burden of Disease Study 2017. Lancet 2018; 393 (10190): 1789-1858. DOl: 10.1016/S01406736(18)32279-7.

2. World Health Organisation. Depression and Other Common Mental Disorders: Global Health Estimates: World Health Organisation; 2017. https://apps.who.int/iris/bitstream/handle/10665/254610/WHO-MSDMER-2017.2-eng.pdf.

3. Kessler RC, Demler O, Frank RG, Olfson M, Pincus HA, Walters EE, et al. Prevalence and treatment of mental disorders, 1990 to 2003. New Engl J Med. 2005;352(24):2515-23.

4. Mokdad AH, Forouzanfar MH, Daoud F, Mokdad AA, El Bcheraoui C, MoradiLakeh $\mathrm{M}$, et al. Global burden of diseases, injuries, and risk factors for young people's health during 1990-2013: A systematic analysis for the Global Burden of Disease Study 2013. Lancet. 2016;387(10036):2383-401. https:// doi.org/10.1016/S0140-6736(16)00648-6.

5. Butler AC, Chapman JE, Forman EM, Beck AT. The empirical status of cognitive-behavioural therapy: a review of meta-analyses. Clin Psychol Rev. 2006;26(1):17-31. https://doi.org/10.1016/j.cpr.2005.07.003.

6. Cuijpers P, van Straten A, Andersson G, van Oppen P. Psychotherapy for depression in adults: a meta-analysis of comparative outcome studies. J Consult Clin Psychol. 2008;76(6):909-22. https://doi.org/10.1037/a0013075. 
7. Fournier JC, DeRubeis RJ, Hollon SD, Dimidjian S, Amsterdam JD, Shelton RC, et al. Antidepressant drug effects and depression severity: a patient-level meta-analysis. JAMA. 2010;303(1):47-53. https://doi.org/10.1 001/jama.2009.1943.

8. Hollon SD, Thase ME, Markowitz JC. Treatment and prevention of depression. Psychol Sci Public Interest. 2002;3(2):39-77. https://doi.org/1 0.1111/1529-1006.00008

9. Kennedy N, Abbott R, Paykel ES. Longitudinal syndromal and sub-syndromal symptoms after severe depression: 10-year follow-up study. Brit J Psychiatry. 2004;184:330-6.

10. Nathan PE, Gorman JM. A guide to treatments that work. 3rd ed. Oxford: Oxford University Press; 2007. https://doi.org/10.1093/med:psych/97801953 04145.001 .0001

11. Vittengl JR, Clark LA, Dunn TW, Jarrett RB. Reducing relapse and recurrence in unipolar depression: a comparative meta-analysis of cognitivebehavioural therapy's effects. J Consult Clin Psychol. 2007;75(3):475-88. https://doi.org/10.1037/0022-006X.75.3.475.

12. Curry J, Silva S, Rohde P, Ginsburg G, Kratochvil C, Simons A, et al. Recovery and recurrence following treatment for adolescent major depression. Arch Gen Psychiatry. 2011;68(3):263-9. https://doi.org/10.1001/archgenpsychia try.2010.150.

13. Wagner CA, Alloy LB, Abramson LY. Trait rumination, depression, and executive functions in early adolescence. J Youth Adolesc. 2015;44(1):18-36. https://doi.org/10.1007/s10964-014-0133-8

14. Zisook S, Lesser I, Stewart JW, Wisniewski SR, Balasubramani GK, Fava M, et al. Effect of age at onset on the course of major depressive disorder. Am J Psychiatry. 2007;164(10):1539-46. https://doi.org/10.1176/appi.ajp.2 007.06101757.

15. Williams JM, Barnhofer T, Crane C, Duggan DS, Shah D, Brennan K, et al. Preadult onset and patterns of suicidality in patients with a history of recurrent depression. J Affect Disord. 2012;138(1-2):173-9. https://doi.org/10.1016/j.ja d.2011.12.011.

16. Rohde P, Lewinsohn PM, Klein DN, Seeley JR, Gau JM. Key Characteristics of Major Depressive Disorder Occurring in Childhood, Adolescence, Emerging Adulthood. Adulthood. Clin Psychol Sci. 2013;1(1). https://doi.org/10.1177/21 67702612457599 .

17. Gladstone TR, Beardslee WR, O'Connor EE. The prevention of adolescent depression. Psychiatr Clin North Am. 2011;34(1):35-52. https://doi.org/10.101 6/j.psc.2010.11.015

18. Lewinsohn PM, Rohde P, Seeley JR, Klein DN, Gotlib IH. Psychosocial functioning of young adults who have experienced and recovered from major depressive disorder during adolescence. J Abnormal Psychol. 2003; 12(3):353-63. https://doi.org/10.1037/0021-843X.112.3.353.

19. Lewinsohn PM, Clarke GN. Psychosocial treatments for adolescent depression. Clin Psychol Rev. 1999;19(3):329-42. https://doi.org/10.1016/s02 72-7358(98)00055-5.

20. Jaffee SR, Moffitt TE, Caspi A, Fombonne E, Poulton R, Martin J. Differences in early childhood risk factors for juvenile-onset and adult-onset depression. Arch Gen Psychiatry. 2002;59(3):215-22. https://doi.org/10.1001/archpsyc. 59.3.215.

21. Johnson D, Dupuis G, Piche J, Clayborne Z, Colman I. Adult mental health outcomes of adolescent depression: A systematic review. Depress Anxiety. 2018:35(8):700-16. https://doi.org/10.1002/da.22777.

22. Fergusson DM, Boden JM, Horwood LJ. Recurrence of major depression in adolescence and early adulthood, and later mental health, educational and economic outcomes. Br J Psychiatry. 2007;191:335-42. https://doi.org/10.11 92/bjp.bp.107.036079.

23. Breedvelt JJF, Kandola A, Kousoulis AA, Brouwer ME, Karyotaki E, Bockting $\mathrm{CLH}$, et al. What are the effects of preventative interventions on major depressive disorder (MDD) in young adults? A systematic review and metaanalysis of randomised controlled trials. J Affect Disord. 2018;239:18-29. https://doi.org/10.1016/j.jad.2018.05.010.

24. Jané-Llopis E, Hosman C, Jenkins R, Anderson P. Predictors of efficacy in depression prevention programmes: Meta-analysis. Brit J Psychiatry. 2003; 183(5):384-97.

25. Merry SN, Hetrick SE, Cox GR, Brudevold-Iversen T, Bir JJ, McDowell H. Psychological and educational interventions for preventing depression in children and adolescents. Cochrane Database Syst Rev. 2011;12:CD003380. https://doi.org/10.1002/14651858.CD003380.pub3.

26. Hetrick SE, Cox GR, Witt KG, Bir נرل, Merry SN. Cognitive behavioural therapy (CBT), third-wave CBT and interpersonal therapy (IPT) based interventions for preventing depression in children and adolescents. Cochrane Database Syst Rev. 2016;8:CD003380. https://doi.org/10.1 002/14651858.CD003380.pub4.

27. Insel T, Cuthbert B, Garvey M, Heinssen R, Pine DS, Quinn K, et al. Research domain criteria (RDoC): toward a new classification framework for research on mental disorders. Am J Psychiatry. 2010;167(7):748-51.

28. Sanislow CA, Pine DS, Quinn KJ, Kozak MJ, Garvey MA, Heinssen RK, et al. Developing Constructs for Psychopathology Research: Research Domain Criteria. J Abnormal Psychology. 2010;119(4):631-9.

29. Natania AC, Alvaro V, Masoud K, Runa B, Kelly AR, David FM, et al. Developing Dimensional, Pandiagnostic Inhibitory Control Constructs With Self-Report and Neuropsychological Data. Assessment. 2018;27:787-802 1073191118754704

30. Zalta AK, Shankman SA. Conducting Psychopathology Prevention Research in the RDoC Era. Clin Psychol Sci Pract. 2016;23:94-104. https://doi.org/1 $0.1111 /$ cpsp. 12144

31. Watkins ER, Roberts H. Reflecting on Rumination: Consequences, causes, mechanisms and treatment of rumination. Behav Res Ther. 2020;127:103573.

32. Watkins ER. Constructive and unconstructive repetitive thought. Psychol Bull. 2008;134(2):163-206. https://doi.org/10.1037/0033-2909.134.2.163.

33. Just $N$, Alloy LB. The response styles theory of depression: tests and an extension of the theory. J Abnorm Psychol. 1997;106(2):221-9. https://doi. org/10.1037//0021-843x.106.2.221.

34. Robinson MS, Alloy LB. Negative Cognitive Styles and Stress-Reactive Rumination Interact to Predict Depression: A Prospective Study. Cogn Ther Res. 2003;27:275-91. https://doi.org/10.1023/A:1023914416469.

35. Nolen-Hoeksema S, Morrow J. A prospective study of depression and posttraumatic stress symptoms after a natural disaster: the 1989 Loma Prieta Earthquake. J Pers Soc Psychol. 1991;61(1):115-21. https://doi.org/10.1037// $0022-3514.61 .1 .115$.

36. Abela JRZ, Brozina K, Haigh EP. An Examination of the Response Styles Theory of Depression in Third- and Seventh-Grade Children: A Short-Term Longitudinal Study. J Abnorm Child Psychol. 2002;30:515-27. https://doi. org/10.1023/A:1019873015594

37. Jones NP, Siegle GJ, Thase ME. Effects of Rumination and Initial Severity on Remission to Cognitive Therapy for Depression. CognTher Res. 2008;32:591. https://doi.org/10.1007/s10608-008-9191-0.

38. Schmaling KB, Dimidjian S, Katon W, Sullivan M. Response styles among patients with minor depression and dysthymia in primary care. J Abnorm Psychol. 2002;111(2):350-6. https://doi.org/10.1037/0021-843X.111.2.350.

39. Spasojević J, Alloy LB. Rumination as a common mechanism relating depressive risk factors to depression. Emotion. 2001;1(1):25-37. https://doi. org/10.1037/1528-3542.1.1.25

40. Hankin BL. Rumination and depression in adolescence: investigating symptom specificity in a multiwave prospective study. J Clin Child Adolesc Psychol. 2008;37(4):701-13. https://doi.org/10.1080/15374410802359627.

41. Nolen-Hoeksema S, Wisco BE, Lyubomirsky S. Rethinking Rumination. Perspect Psychol Sci. 2008;3(5):400-24. https://doi.org/10.1111/j.1745-6924.2 008.00088.

42. Shaw ZA, Hilt LM, Starr LR. The developmental origins of ruminative response style: An integrative review. Clin Psychol Rev. 2019;74:101780. https://doi.org/10.1016/j.cpr.2019.101780.

43. Fair DA, Cohen AL, Dosenbach NU, Church JA, Miezin FM, Barch DM, et al. The maturing architecture of the brain's default network. Proc Nat Acad Sci. 2008:105(10):4028-32. https://doi.org/10.1073/pnas.0800376105.

44. Sherman LE, Rudie JD, Pfeifer JH, Masten CL, McNealy K, Dapretto M. Development of the default mode and central executive networks across early adolescence: A longitudinal study. Dev Cogn Neurosci. 2014;10:148-59. https://doi.org/10.1016/j.den.2014.08.002.

45. Berman MG, Peltier S, Nee DE, Kross E, Deldin PJ, Jonides J. Depression, rumination and the default network. Soc Cogn Affect Neurosci. 2011;6(5): 548-55.

46. Bessette KL, Jenkins LM, Skerrett KA, Gowins JR, DelDonno SR, Zubieta J-K, et al. Reliability, Convergent Validity and Time Invariance of Default Mode Network Deviations in Early Adult Major Depressive Disorder. Front Psychiatry. 2018;9:244

47. de Kwaasteniet BP, Rive MM, Ruhe $H G$, Schene AH, Veltman DJ, Fellinger L, et al. Decreased Resting-State Connectivity between Neurocognitive Networks in Treatment Resistant Depression. Front Psychiatry. 2015;6:28.

48. Burkhouse $\mathrm{KL}$, Jacobs $\mathrm{RH}$, Peters AT, Ajilore $\mathrm{O}$, Watkins $\mathrm{E}$, Langenecker SA Neural Correlates of Rumination in Adolescents with Remitted Major 
Depressive Disorder and Healthy Controls. Cogn Affect Behav Neurosci. 2016:17:394-405.

49. Sheline YI, Price JL, Yan Z, Mintun MA. Resting - state functional MRI in depression unmasks increased connectivity between networks via the dorsal nexus. Proc Nat Acad Sci U S A. 2010;107(24):11020-5. https://doi. org/10.1073/pnas. 1000446107.

50. Cooney RE, Joormann J, Eugene F, Dennis EL, Gotlib IH. Neural correlates of rumination in depression. Cogn Affect Behav Neurosci. 2010;10(4):470-8. https://doi.org/10.3758/CABN.10.4.470.

51. Farb NA, Anderson AK, Bloch RT, Segal ZV. Mood - linked responses in medial prefrontal cortex predict relapse in patients with recurrent unipolar depression. Biol Psychiatry. 2011;70(4):366-72. https://doi.org/10.1016/j. biopsych.2011.03.009.

52. Johnson MK, Nolen-Hoeksema S, Mitchell KJ, Levin Y. Medial cortex activity, self-reflection and depression. Soc Cogn Affect Neurosci. 2009;4(4):313-27. https://doi.org/10.1093/scan/nsp022.

53. Siegle GJ, Hasselmo ME. Using connectionist models to guide assessment of psychological disorder. Psychol Assessment. 2002;14(3):263-78. https:// doi.org/10.1037/1040-3590.14.3.263.

54. Hamilton JP, Furman DJ, Chang C, Thomason ME, Dennis E, Gotlib IH. Default-Mode and Task- Positive Network Activity in Major Depressive Disorder: Implications for Adaptive and Maladaptive Rumination. Biol Psychiatry. 2011;70(4):327-33. https://doi.org/10.1016/j.biopsych.2011.02.003.

55. Schiller CE, Minkel J, Smoski MJ, Dichter GS. Remitted major depression is characterized by reduced prefrontal cortex reactivity to reward loss. J Affect Disord. 2013;151(2):756-62. https://doi.org/10.1016/j.jad.2013.06.016.

56. Thomas EJ, Elliott R, McKie S, Arnone D, Downey D, Juhasz G, et al. Interaction between a history of depression and rumination on neural response to emotional faces. Psychol Med. 2011;41(9):1845-55. https://doi. org/10.1017/S0033291711000043.

57. Zhu X, Wang X, Xiao J, Liao J, Zhong M, Wang W, et al. Evidence of a dissociation pattern in resting-state default mode network connectivity in first - episode,treatment -naïve major depression patients. Biol Psychiatry. 2012;71(7):611-7. https://doi.org/10.1016/j.biopsych.2011.10.035.

58. Hamilton JP, Chen G, Thomason ME, Schwartz ME, Gotlib IH. InvestigatingneuralprimacyinMajorDepressiveDisorder: multivariateGrangercausalityanalysisofresting-statefMRltime-seriesdata. Mol Psychiatry. 2011;16(7):763-72. https://doi.org/10.1038/mp.2010.46.

59. Langenecker SA, Jacobs RH, Passarotti AM. Current Neural and Behavioral Dimensional Constructs across Mood Disorders. Curr Behav Neurosci Rep. 2014;:1114-53.

60. Langenecker SA, Mickey BJ, Eichhammer P, Sen S, Elverman KH, Kennedy SE, et al. Cognitive control as a 5-ht1a-based domain that is disrupted in major depressive disorder. Front Psychol. 2019;10:691.

61. Stange JP, Jenkins LM, Hamlat EJ, Bessette KL, DelDonno SR, Kling LR, et al. Disrupted engagement of networks supporting hot and cold cognition in remitted major depressive disorder. J Affect Disord. 2017;227:183-91.

62. Jacobs RH, Jenkins LM, Gabriel LB, Barba A, Ryan KA, Weisenbach SL, et al. Increased Coupling of Intrinsic Networks in Remitted Depressed Youth Predicts Rumination and Cognitive Control. PloS One. 2014;9:e104366. https://doi.org/10.1371/journal.pone.0104366.

63. Roberts H, Watkins ER, Wills AJ. Cueing an unresolved personal goal causes persistent ruminative self-focus: An experimental evaluation of control theories of rumination. J Behav Ther Exp Psychiatry. 2013;44(4):449-55. https://doi.org/10.1016/j.jbtep.2013.05.004

64. Spinhoven P, Klein N, Kennis M, Cramer AOJ, Siegle G, Cuijpers P, et al. The effects of cognitive-behaviour therapy for depression on repetitive negative thinking: A meta-analysis. Behav Res Ther. 2018;106:71-85. https://doi.org/1 0.1016/j.brat.2018.04.002.

65. Watkins ER, Mullan E, Wingrove J, Rimes K, Steiner H, Bathurst N, et al. Rumination-focused cognitive-behavioural therapy for residual depression: phase II randomised controlled trial. Br J Psychiatry. 2011;199(4):317-22. https://doi.org/10.1192/bjp.bp.110.090282.

66. Teismann T, von Brachel R, Hanning S, Grillenberger M, Hebermehl L, Hornstein I, et al. A randomised controlled trial on the effectiveness of a rumination-focused group treatment for residual depression. Psychother Res. 2014;24(1):80-90. https://doi.org/10.1080/10503307.2013.821636.

67. Topper M, Emmelkamp PM, Watkins E, Ehring T. Prevention of anxiety disorders and depression by targeting excessive worry and rumination in adolescents and young adults: A randomised controlled trial. Behav Res Ther. 2017;90:123-36. https://doi.org/10.1016/j.brat.2016.12.015.
68. Cook L, Mostazir M, Watkins E. Reducing Stress and Preventing Depression (RESPOND): Randomised Controlled Trial of Web-Based Rumination-Focused Cognitive Behavioural Therapy for High-Ruminating University Students. J Med Internet Res. 2019;21(5):e11349. https://doi.org/10.2196/11349.

69. Jacobs RH, Watkins ER, Peters AT, Feldhaus CG, Barba A, Carbray J, et al. Targeting Ruminative Thinking in Adolescents at Risk for Depressive Relapse: Rumination-Focused Cognitive Behaviour Therapy in a Pilot Randomised Controlled Trial with Resting State fMRI. PLoS One. 2016;11(11): e0163952. https://doi.org/10.1371/journal.pone.0163952.

70. Schleider JL, Weisz JR. Reducing risk for anxiety and depression in adolescents: Effects of a single-session intervention teaching that personality can change. Behav Res Ther. 2016;87:170-81. https://doi.org/10.1 016/j.brat.2016.09.011.

71. American Psychiatric Association. Diagnostic and Statistical Manual of Mental Disorders. 5th ed. Arlington: American Psychiatric Association; 2013.

72. Kaufman J, Birmaher B, Brent D, Rao U, Flynn C, Moreci P, et al. Schedule for affective disorders and schizophrenia for school-age children-present and lifetime version (K-SADS-PL): initial reliability and validity data. J Am Acad Child Adolesc Psychiatry. 1997;36(7):980-8. https://doi.org/10.1097/00004 583-199707000-00021.

73. Kaufman J, Schweder AE. The Schedule for Affective Disorders and Schizophrenia for School-age Children: Present and Lifetime Version (KSADS-PL). In: Hersen M, editor. Comprehensive Handbook of Psychological Assessment, Personality Assessment, vol. 2. Hoboken: Wiley; 2004. p. 247-55.

74. Treynor W, Gonzalez R, Nolen-Hoeksema S. Rumination Reconsidered: A Psychometric Analysis. Cogn Ther Res. 2003;27(3):247-59. https://doi.org/1 0.1023/A:1023910315561.

75. Wechsler D. Wechsler Abbreviated Scale of Intelligence-Second Edition (WASI-II). San Antonio: NCS Pearson; 2011

76. Poznanski E, Mokros H. Children's Depression Rating Scale-Revised (CDRS-R). Los Angeles: WPS; 1996.

77. Linehan MM, Comtois K. Lifetime Parasuicide History. Seattle: University of Washington; 1996. Unpublished work

78. Watkins ER. Rumination-Focused Cognitive-Behavioural Therapy for Depression. Guildford: Guildford Press; 2016.

79. Watkins ER, Taylor RS, Byng R, Baeyens C, Read R, Pearson K, et al. Guided self-help concreteness training as an intervention for major depression in primary care: a Phase II randomized controlled trial. Psychol Med. 2012:42(7): 1359-71. https://doi.org/10.1017/S0033291711002480.

80. Martell CR, Addis ME, Jacobson NS. Depression in Context: Strategies for Guided Action. New York: W. W. Norton \& Company, Inc.; 2001.

81. Jacobson NS, Martell CR, Dimidjian S. Behavioural activation treatment for depression: returning to contextual roots. Clin Psychol Sci Pract. 2001;8:25570. https://doi.org/10.1093/clipsy.8.3.255.

82. Gollwitzer PM. Implementation intentions: Strong effects of simple plans. Am Psychol. 1999;54(7):493-503. https://doi.org/10.1037/0003-066X.54.7.493.

83. Gollwitzer PM, Sheeran P. Implementation intentions and goal achievement: A meta-analysis of effects and processes. In: Zanna MP, editor. Advances in experimental social psychology: Advances in experimental social psychology, vol. 38: Elsevier Academic Press; 2006. p. 69-119. https://doi. org/10.1016/50065-2601(06)38002-1.

84. Young J, Beck AT. Cognitive Therapy Scale Rating Manual. Cambridge: The Beck Institute; 1980.

85. Whitmer A, Gotlib IH. Brooding and reflection reconsidered: A factor analytic examination of rumination in currently depressed, formerly depressed, and never depressed individuals. Cogn Ther Res. 2011;35(2):99107. https://doi.org/10.1007/s10608-011-9361-3.

86. Speer DC. Clinically significant change: Jacobson and Truax (1991) revisited. J Consult Clin Psychol. 1992;60(3):402. https://doi.org/10.1037//0022-006x. 60.3.402.

87. Stange JP, Bessette $K L$, Jenkins $L M$, Burkhouse $K L$, Peters AT, Feldhaus C, et al. Attenuated Intrinsic Connectivity within Cognitive Control Network Among Individuals with Remitted Depression: Temporal Stability and Association with Negative Cognitive Styles. Hum Brain Map. 2017;38:2939-54.

88. Nolen-Hoeksema S, Morrow J. Effects of rumination and distraction on naturally occurring depressed mood. Cogn Emotion. 1993;7(6):561-70. https://doi.org/10.1080/02699939308409206.

89. Harris PA, Taylor R, Thielke R, Payne J, Gonzalez N, Conde JG. Research electronic data capture (REDCap)--a metadata-driven methodology and workflow process for providing translational research informatics support. J Biomed Inform. 2009;42(2):377-81. https://doi.org/10.1016/j.jbi.2008.08.010. 
90. Health Insurance Portability and Accountability Act 1996. Available from: https://www.govinfo.gov/content/pkg/BILLS-104s1028is/pdf/BILLS-104s102 8is.pdf. Accessed 15 Jan 2020.

91. General Data Protection Regulation 2018. Available from: https:/gdpr-info.eu/. Accessed 15 Jan 2020.

92. Angrist JD, Imbens GW, Rubin DB. Identification of Causal Effects Using Instrumental Variables. J Am Stat Assoc. 1996;91:444-55. https://doi.org/10.1 080/01621459.1996.10476902.

93. Dunn G, Maracy M, Tomenson B. Estimating treatment effects from randomised clinical trials with noncompliance and loss to follow-up: the role of instrumental variable methods. Stat Methods Med Res. 2005;14:36995. https://doi.org/10.1191/0962280205sm403oa.

\section{Publisher's Note}

Springer Nature remains neutral with regard to jurisdictional claims in published maps and institutional affiliations.

Ready to submit your research? Choose BMC and benefit from:

- fast, convenient online submission

- thorough peer review by experienced researchers in your field

- rapid publication on acceptance

- support for research data, including large and complex data types

- gold Open Access which fosters wider collaboration and increased citations

- maximum visibility for your research: over $100 \mathrm{M}$ website views per year

At $\mathrm{BMC}$, research is always in progress.

Learn more biomedcentral.com/submissions 